\title{
Sistemi insediativi ed organizzazione del territorio nel Bronzo recente dell'Alto Adige ${ }^{1}$ \\ Umberto Tecchiati, † Massimiliano Di Pillo
}

\section{L'areale}

Il territorio altoatesino, comprendente essenzialmente l'alto bacino dell'Adige, con il suo affluente principale, l'Isarco, presenta caratteri schiettamente alpini, con vette che sfiorano i $4.000 \mathrm{~m}$ di quota, vasti altipiani ed una capillare rete di valli che svolgono un'importante funzione di collegamento, non solo a livello locale, ma anche su scala europea.

Preminenti, per ampiezza e rilevanza nelle comunicazioni, sono il solco vallivo dell'Adige, naturalmente, distinguibile in un tratto iniziale, a decorso prevalentemente longitudinale a partire dal Passo di Resia (Val Venosta), e in uno medio, a valle della conca di Merano (Val d'Adige propriamente detta, fino alla Chiusa di Rivoli, nelle Prealpi veronesi), nettamente trasversale; la Val d'Isarco, che origina dal Passo del Brennero e, dopo un andamento trasversale, s'innesta nella prima all'altezza di Bolzano; la Valle della Rienza, longitudinale, che confluisce nella Val d'Isarco all'altezza di Bressanone e rappresenta un agevole collegamen-

1 Il presente contributo rappresenta l'edizione, tale e quale salvo l'apparato critico e piccole aggiunte o correzioni resesi nel frattempo necessarie a causa dell'avanzamento degli studi, di un contributo portato da me e dal compianto Massimiliano di Pillo al workshop organizzato nel giugno 2002 al Museo Archeologico Nazionale di Parma in previsione del congresso nazionale sull'età del Bronzo recente in Italia tenuto in autunno dello stesso anno a Lido di Camaiore (Сосchi Genick 2004). Riproporlo qui significa in primo luogo rendere omaggio a uno studioso, che già troviamo nella lista degli autori di "Ladinia" (Di PILlo 1999), scomparso nel fiore della giovinezza, che molto già aveva, e certo molto di più avrebbe contribuito in futuro al progresso delle conoscenze sulla protostoria dell'area alpina atesina.

Ladinia XXIX (2005), 7-23

ISSN 1124-1004; (C) Istitut Ladin “Micurà de Rü”, San Martin de Tor (BZ). 


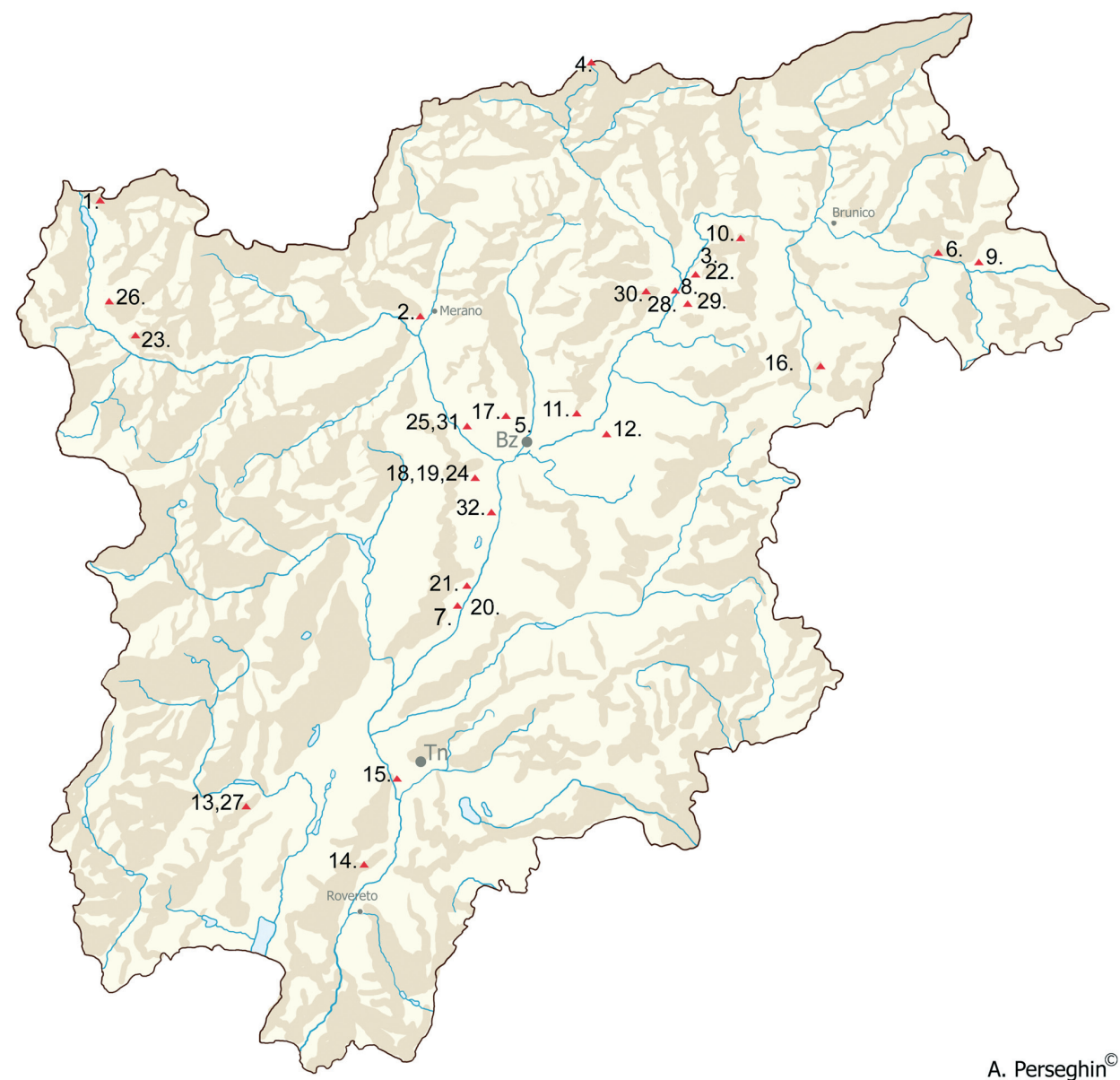

Fig. 1: Carta di distribuzione dei siti citati nel testo (in ordine alfabetico). Elaborazione per la stampa a cura di Alessia Perseghin

to tra questa ed il bacino della Drava, affluente del Danubio, attraverso la quasi impercettibile Sella di Dobbiaco. ${ }^{2}$

Carattere comune di queste vallate (come di quelle minori) è la presenza di salti di quota, spesso associati a restringimenti del piano vallivo (chiuse), quando non a vere e proprie gole, soprattutto nel loro tratto terminale: ne sono esempio la Rienza, poco prima della confluenza nell'Isarco, e lo stesso Isarco, in prossimità della conca di

2 Il passaggio tra le valli della Rienza e della Drava è così sfumato, che entrambe (la Drava limitatamente all'alto corso, fino alla conca di Lienz, nel Tirolo dell'Est) sono comprese sotto il nome di Val Pusteria. 
1. Passo di Resia/Reschenpass

2. Merano/Meran

3. Valle dell'Isarco/Eisacktal

4. Passo del Brennero/Brennerpass

5. Bolzano/Bozen

6. Valle della Rienza (Pusteria)/ Rienztal (Pustertal)

7. Valle dell'Adige/Etschtal

8. Bressanone-Cassianeum/ Brixen-Cassianeum

9. Dobbiaco/Toblach

10. Rodengo/Rodeneck

11. Renòn/Ritten

12. Fiè-Castelrotto/Völs-Kastelruth

13. Fiavè/Gustinacci

14. Nomi-Cef

15. Trento-Groa

16. Badia-Sotćiastel

17. San Genesio-Groaßer Knott/ Jenesien-Groaßer Knott

18. Appiano-Siechenhaus (Gamberoni)/ Eppan-Siechenhaus (Gamberoni)

19. Appiano-Wildermannbühel/

Eppan-Wildermannbühel
20. Favogna/Fennhals

21. Cortaccia/Kurtatsch

22. Varna-Nössing/Vahrn-Nössing

23. Sluderno-Ganglegg/Schluderns-Ganglegg

24. Appiano-Putzer Gschleier/ Eppan-Putzer Gschleier

25. Terlano-Settequerce/Terlan-Siebeneich

26. Malles-Margun Egg/Mals-Margun Egg

27. Fiavè-Carera

28. Bressanone-Via Castellano/BrixenKöstlanerstrasse

29. Bressanone-Albanbühel/Brixen-Albanbühel

30. Bressanone-Via Monte Ponente/ Brixen-Pfeffersberg

31. Settequerce-Via Steuer/ Siebeneich-Steuerstrasse

32. Vadena-Laimburg/Pfatten-Laimburg

Bolzano. Ciò, unito al corso irregolare dei fiumi e al loro regime incostante, rendeva certamente problematica la percorribilità dei fondivalle, sicché, soprattutto nelle zone appena citate, appare possibile che, almeno in alcuni periodi, fossero preferiti percorsi a mezzacosta o attraverso gli altipiani (Rodengo, Renòn e Fiè-Castelrotto).

Prescindendo dalle frequentazioni mesolitiche, documentate soprattutto ad alta quota, la colonizzazione neolitica è relativamente precoce (VI-V millennio a.C.): particolarmente preferiti appaiono gli stanziamenti sui terrazzi orografici di media quota (m 600-900 circa), caratterizzati da clima ancora abbastanza mite, deboli pendenze e suoli leggeri e discretamente produttivi. 


\section{Il caso di studio: profilo cronologico-culturale}

Il Bronzo recente costituisce, in Trentino-Alto Adige, il momento in cui sembrano consolidarsi le spinte, già presenti nella fase terminale del Bronzo medio, verso la formazione di un'entità culturale autonoma (per lo meno in senso relativo), perdurante fino a tutta l'età del Ferro e coinvolgente, a seconda dei periodi e con una dinamica non del tutto chiara, aree nordalpine austriache e svizzere.

Per quanto alcune zone, soprattutto nord-orientali, dell' Alto Adige manifestino, già dal Bronzo antico, una certa lontananza dagli aspetti culturali in senso lato palafitticoli, è con la fase, definita a suo tempo da Renato PerINI, Fiavè $7^{\circ},{ }^{3}$ corrispondente, a nostro giudizio, alla fine del Bronzo medio ed all'inizio del Bronzo recente, che si giunge ad una prima affermazione, a livello regionale, di elementi locali; successivo all'aspetto citato, o di Gustinaci, ma ancora databile entro il Bronzo recente 1 (o Bz D della cronologia mitteleuropea), è un complesso ceramico, a dir il vero un po' scarso, da Nomi-Cef ${ }^{4}$ e Trento-Groa, strato $C,{ }^{5}$ che rivela significativi agganci con la successiva facies di Luco (fase A), il cui inizio può collocarsi certamente nel Bronzo recente 2 (prima parte dell'Ha A1), ma la cui articolazione in sottofasi distinte presenta ancora numerosi problemi.

I siti citati sono tutti situati in Trentino: materiali analoghi sono presenti, seppure in misura limitata ed in contesti talora d'incerta stratigrafia, anche in Alto Adige, come a Badia-Sotćiastel, ${ }^{6}$ San Genesio-Groaßer Knott, ${ }^{7}$ Appiano-Siechenhaus. ${ }^{8}$

L'autonomia acquisita dalla regione atesina, tra la fine del Bronzo medio e l'inizio del finale, non coinvolge, ovviamente, tutte le sfere culturali: la metallurgia mantiene stretti collegamenti con la facies palafitticola fino al crollo di questa, ed anche durante il Bronzo finale emergono significativi contatti tra la produzione atesina e quella di aree, sia pur limitate, dell'Italia settentrionale,

3 Cf. Perini 1984, 1987, 1994.

4 Cf. Marzatico 1985-1986.

5 Cf. PERINI 1979.

6 Cf. Tecchiati 1998a.

7 Cf. Niederwanger/Tecchiati 2000.

8 Per il sito di Appiano - Giardineria Gamberoni (Siechenhaus) si veda la monografia di LeITNER 1988. Per l'area di Appiano in generale dalla preistoria all'alto Medioevo cf. Lunz 1990; sistemi insediativi nell'Oltradige: Di Pillo 2002; ancora sui sistemi insediativi tra Bronzo finale e prima età del Ferro cf. Alberti et al. 2005 (in stampa). 
come le zone alpine e prealpine venete e lombarde ed il "gruppo" di Fontanella Mantovana. Anche sul piano socioeconomico ed ideologico, per quanto taluni aspetti sembrino pressoché specifici dell'area, fattori di più ampia integrazione sembrerebbe, ad esempio, il diffondersi delle pratiche funerarie crematorie.

Del resto, qualche contatto con l'area padana è ravvisabile anche nella presenza, isolata, di anse cilindrorette (il caso di Appiano-Wildermannbühel pare certo), e nell'attestazione di ceramica decorata "a turbante", per lo meno nel Trentino meridionale; ${ }^{9}$ né si può trascurare l'evidente connessione tra gli orli a tesa a spigolo vivo della ceramica Luco A e di quella veneta del Bronzo recente evoluto, a suo tempo inquadrata da G. Leonardi, entrambi con chiare assonanze nella ceramica dei Campi d'Urne.

Un ulteriore potente legante cronologico tra gli esiti formali del Bronzo recente evoluto veneto e i corrispettivi altoatesini, si ha per esempio a Custoza di Sommacampagna, ${ }^{10}$ dove compare, in un contesto anche simbolico in fondo non dissimile dalle coeve manifestazioni tipicamente alpine (Brandopferplätze), una decorazione a onde incise che trova puntuali riscontri a Seeberg nelle Alpi Sarentine e al Groaßer Knott di San Genesio.

\section{Storia delle ricerche e stato delle fonti}

La ricerca territoriale ha, nell'Alto Adige, una lunga storia: già negli ultimi decenni dell'Ottocento, infatti, si segnalano i primi studi, ad opera principalmente di eruditi locali; ma è con il Novecento che s'intensificano le ricerche, ad opera soprattutto di Adrian Egger, attivo fino agli anni Quaranta, e Georg Innerebner, la cui Wallburgenstatistik segna il secondo ed il terzo quarto del secolo.

L'opera di Innerebner, per quanto infaticabile e minuziosa, ed arricchita negli ultimi anni dal controllo scientifico di Reimo Lunz, presenta, nondimeno, ampie lacune: da un lato, in quanto, come lascia intendere il termine Wallburg, rivolta

9 Recenti scavi a Laion (per i quali si vedano, sinteticamente, ATTARDo/BAnZI/TeCCHIATI 2004) mostrano peraltro che la decorazione a finta torsione di carene di recipienti fini (ciotole), già presente ad Appiano - Siechenhaus (DAL RI 1990) risale la Val d'Isarco almeno fino all'imbocco della Val Gardena.

${ }^{10}$ Cf. SALZANi 1996-1997. Il reperto è quello rappresentato in Fig. 4 e alla Tav. IX.26; decorazione affine, ma meno complessa (una sola fila di onde o zig zag) è nel reperto di Tav. VII.13; potenzialmente affine, inoltre, il reperto a Tav. XI.38, tutti da US 25. Il reperto di Tav. II.3, da US 50, è ben confrontabile con il coccio pubblicato da Niederwanger/Tecchiati 2000, Fig. 55, 1 (dal Groaßer Knott) e 55.2 (da Seeberg). 
essenzialmente alla scoperta ed all'analisi dei soli siti d'altura; dall'altro, perché priva d'una solida base cronologica e non sempre attendibile.

Il passaggio delle competenze in materia di beni culturali dallo Stato alle Province Autonome di Trento e Bolzano, nel 1973-1974, ha portato a un più attivo intervento istituzionale e a più efficaci controlli delle attività edilizie e infrastrutturali. Il quadro attualmente a disposizione, per quanto ricco dal punto di vista della "rappresentatività numerica", e privo di grandi lacune geografiche, appare ancora notevolmente provvisorio per la scarsa definizione cronologica di molti siti, in buona parte sostanzialmente inediti: nella presente sede, perciò, s'è preferito operare un'analisi più grossolana, di taglio sostanzialmente diacronico, con riferimenti anche all'evidenza dal Trentino.

\section{Caratteri insediativi e dinamica del popolamento}

Nel corso dell'età del Bronzo, come noto, s'assiste al progressivo stabilizzarsi dell'insediamento, ${ }^{11}$ in tutto il nostro Paese; in area alpina, il fenomeno è accompagnato dalla crescente presa di possesso degli ambiti montani, sia con insediamenti stabili, sia con siti temporanei, probabilmente adibiti allo svolgimento di determinate attività, di cui le più importanti erano certamente quelle pastorali e minerarie.

In Alto Adige, si contano, ${ }^{12}$ tra sicuri e presunti, 82 siti per il Bronzo antico, 90 per il Bronzo medio, 85 per il Bronzo recente 1 (nel senso di cui sopra) e 80 per il Luco A (Fig. 2). Il numero sostanzialmente costante può essere spiegato, senza dubbio, con la notevole continuità insediativa cui s'è fatto cenno, condizionata in area alpina anche dalla natura soli; ma d'altra parte, non si può escludere che, in una certa misura, esso sia dovuto alla scarsa definizione cronologica di molti complessi.

A fronte di una generale continuità insediativa (i siti di nuova fondazione non superano, dopo la modesta cesura tra Bronzo antico e Bronzo medio, il 35\% sul totale: Fig. 3), due aspetti sembrano particolarmente interessanti: in primo luogo,

${ }^{11} \mathrm{Cf}$. con riferimento all'area in esame, le considerazioni tracciate da TECCHIATI 2005 (in stampa).

${ }^{12}$ Il conteggio si riferisce al censimento stilato dagli autori nel 2000. Esso si rifà, a sua volta, alle liste compilate da Massimiliano Di Pillo nella sua tesi di dottorato (Di Pillo 1991-1994). Benché si debba sottolineare che alcuni siti si sono aggiunti, si può ritenere che il peso relativo della loro ripartizione in senso cronologico non sia sostanzialmente mutato. 


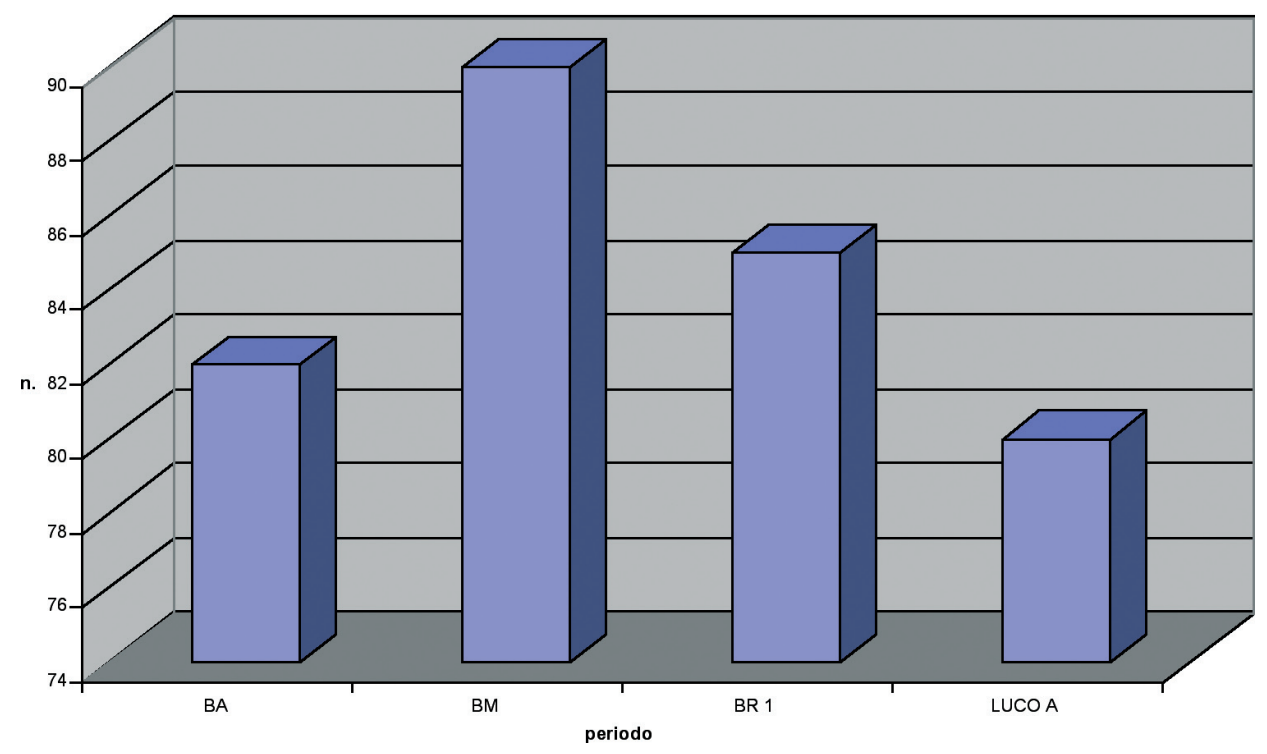

Fig. 2: Frequenza dei siti nel corso dell'età del Bronzo (BA= Bronzo antico; $B M=$ Bronzo medio; BR: Bronzo recente)

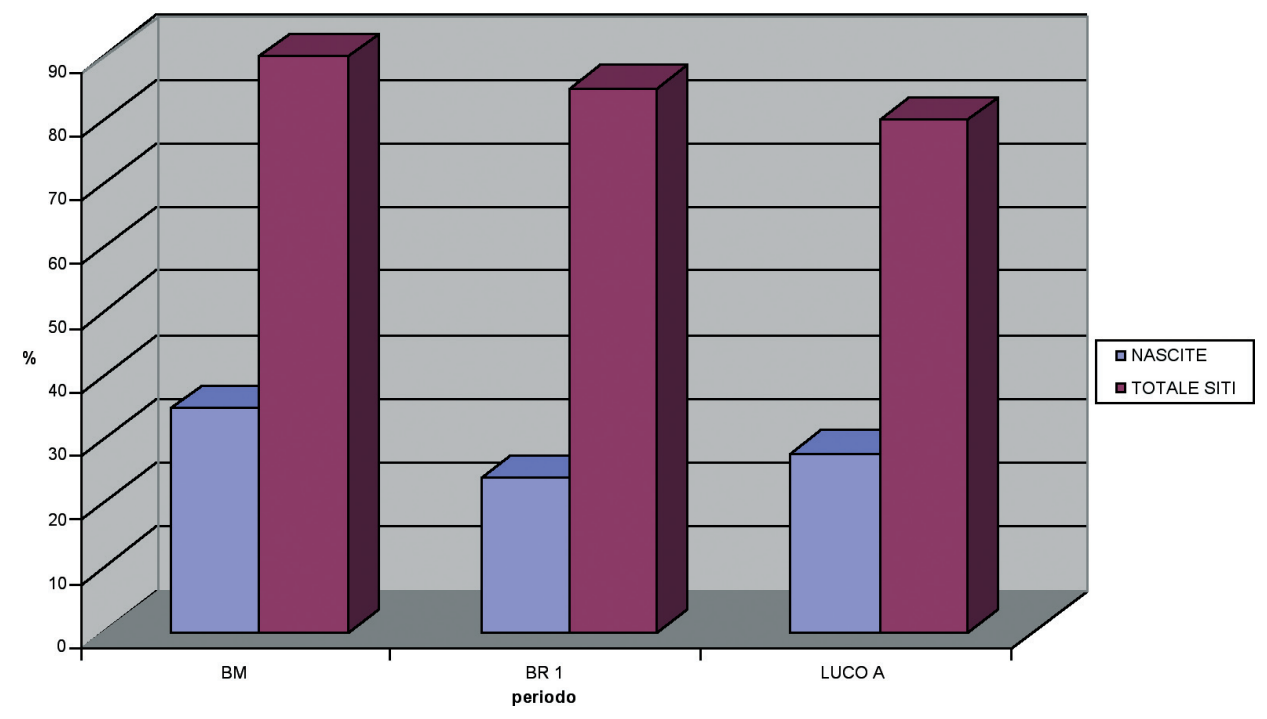

Fig. 3: Bilancia insediativa (BM= Bronzo medio; $B R$ : Bronzo recente) 


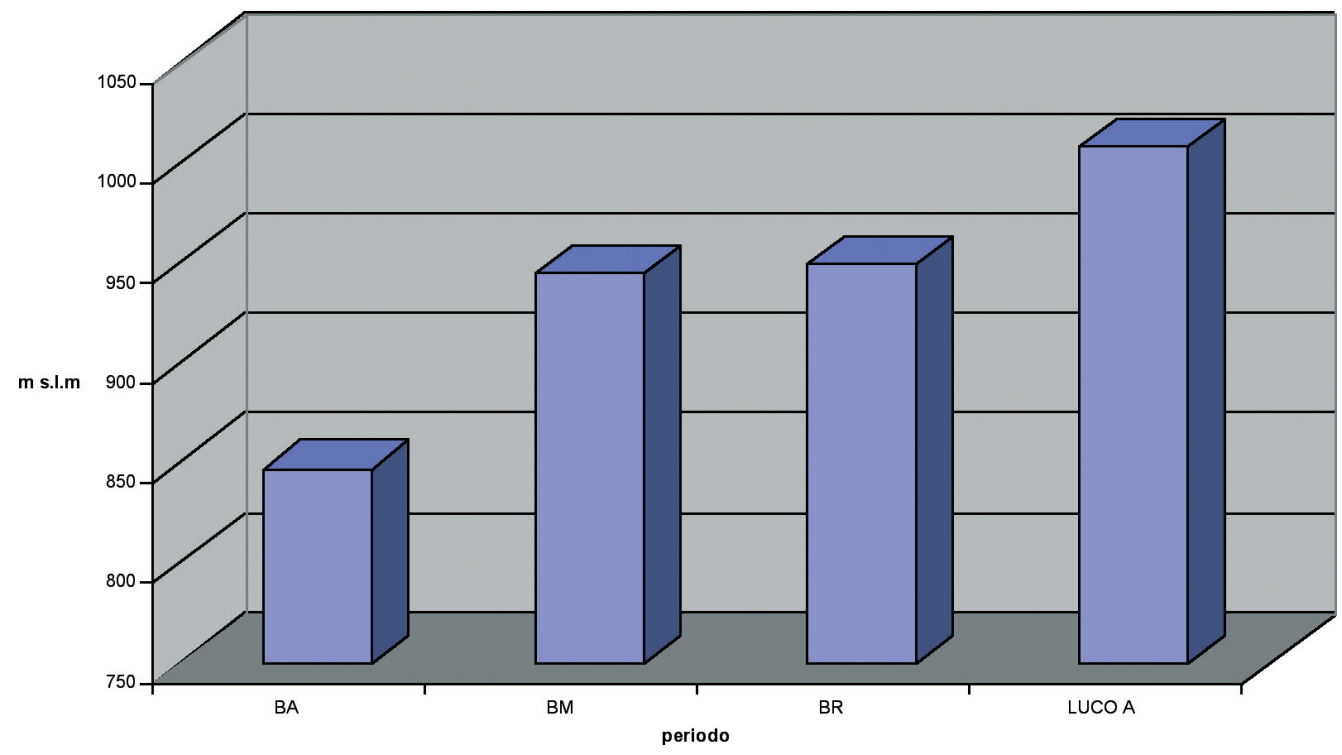

Fig. 4: Quota media degli insediamenti ( $\mathrm{BA}=$ Bronzo antico; $\mathrm{BM}=$ Bronzo medio; BR: Bronzo recente)

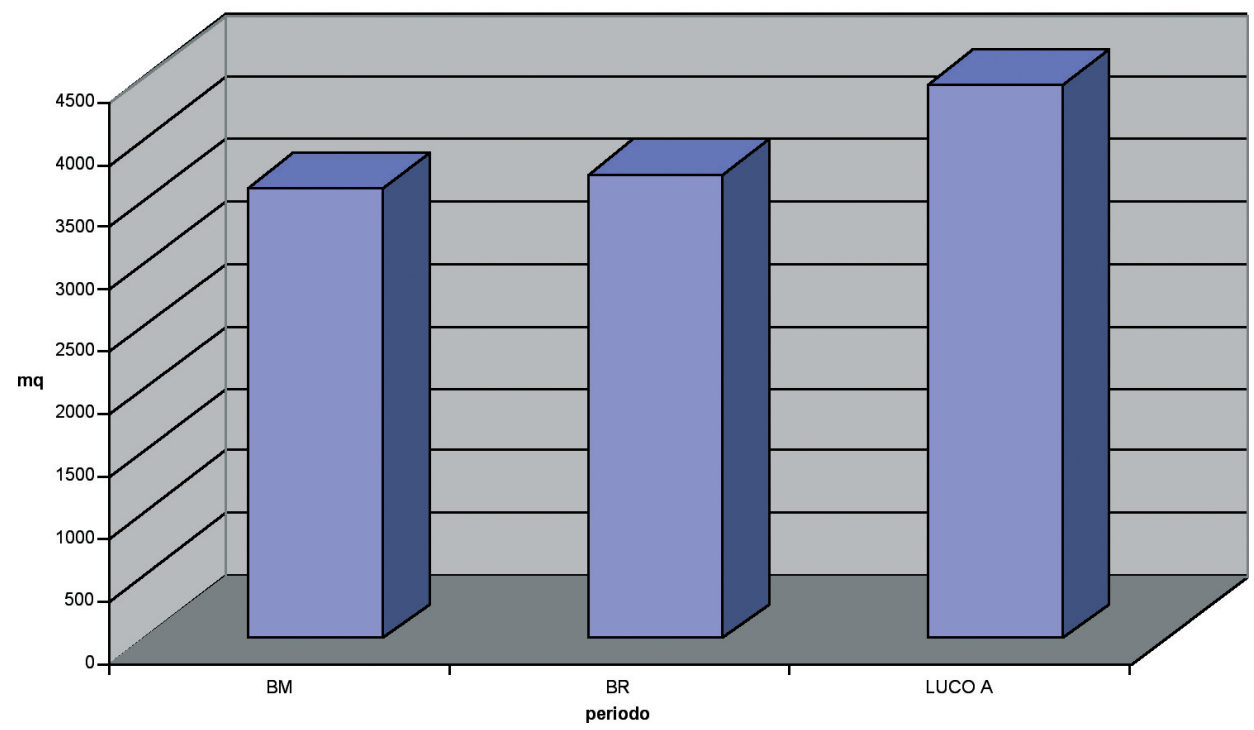

Fig. 5: Aumento della superficie media degli insediamenti (BM= Bronzo medio; BR: Bronzo recente) 
l'incremento della quota media dei siti (Fig. 4) ${ }^{13}$, in secondo, il loro aumento dimensionale (Fig. 5) ${ }^{14}$.

Il primo fenomeno sembrerebbe interpretabile nel senso di una precisa scelta insediativa, volta allo sfruttamento delle risorse dei piani montani, forse anche su iniziativa dei gruppi egemoni: esso, tra l'altro, sembra più precoce in Trentino, per quanto in quest'area la ricerca territoriale sia, forse, più indietro e, dunque, l'osservazione necessiti di ulteriori controlli.

L'aumento delle dimensioni medie dei siti, parallelamente alla diminuzione numerica degli stessi a partire dal Bronzo recente 1, sembra testimoniare, invece, un processo di selezione e concentrazione dell'abitato, ancorché in misura neppure lontanamente paragonabile a quella riscontrata, più o meno nello stesso periodo, in altre aree italiane: ${ }^{15}$ ciò può essere legato al progressivo costituirsi di comunità più ampie ed alla formazione di sistemi insediativi relativamente complessi, come si cercherà di mostrare in seguito.

Il crescente interesse per le risorse dei piani montani è testimoniato anche dall'utilizzazione presunta dei suoli in prossimità degli abitati: ${ }^{16}$ la Fig. 6 mostra come, tra il Bronzo medio ed il Luco A, a fronte di una sostanziale stabilità delle superfici adibite a coltivo, vi sia un netto incremento delle aree tenute a prato e pascolo, con corrispondente riduzione delle zone boscate. ${ }^{17}$

Ciò sembra trovare conferma, peraltro, nel diffondersi, sempre più accentuato nei diagrammi pollinici, di testimonianze di deforestazione e vegetazione prativa.

13 A titolo di confronto, va ricordato che la quota media dei Comuni dell'Alto Adige, prima del riordinamento del 1928-1929, era di circa 959 m (quella attuale ammonta approssimativamente a $851 \mathrm{~m}$ ).

${ }^{14}$ Il calcolo delle dimensioni dei siti presenta numerose difficoltà per la natura di molti rilievi e per l'esistenza di insediamenti su terrazzo non distinto, versante, conoide o fondovalle: l'adozione d'un metodo di calcolo coerente, ad ogni modo, conferisce una qualche significatività ai dati qui esposti.

${ }^{15}$ Il dato, però, è probabilmente sottostimato, in quanto, nel calcolo, non si sono potuti inserire gli abitati di conoide, che conoscono, come si vedrà avanti, un deciso incremento con la tarda età del bronzo e che, a giudicare dagli sviluppi successivi, appaiono tra i più significativi ed importanti.

${ }^{16}$ Quest'analisi s’è basata, in mancanza di sufficienti dati paleoambientali, sulle carte d'utilizzazione del suolo, redatte tra gli anni Cinquanta e Sessanta, a cura del CNR e della Direzione Generale del Catasto: con tale documentazione, in effetti, sarebbe più prudente parlare, più che d'utilizzo in antico, di vocazione d'un certo territorio. È però vero che, ove presenti, i dati archeozoologici, paleobotanici e paleoambientali sembrano sostenere l'evidenza attuale, in una regione che, dal punto di vista ecologico, appare generalmente ben conservata (si veda subito avanti).

17 Anche questo fenomeno appare più precoce in Trentino, dove raggiunge il suo culmine nel Bronzo recente 1 . 


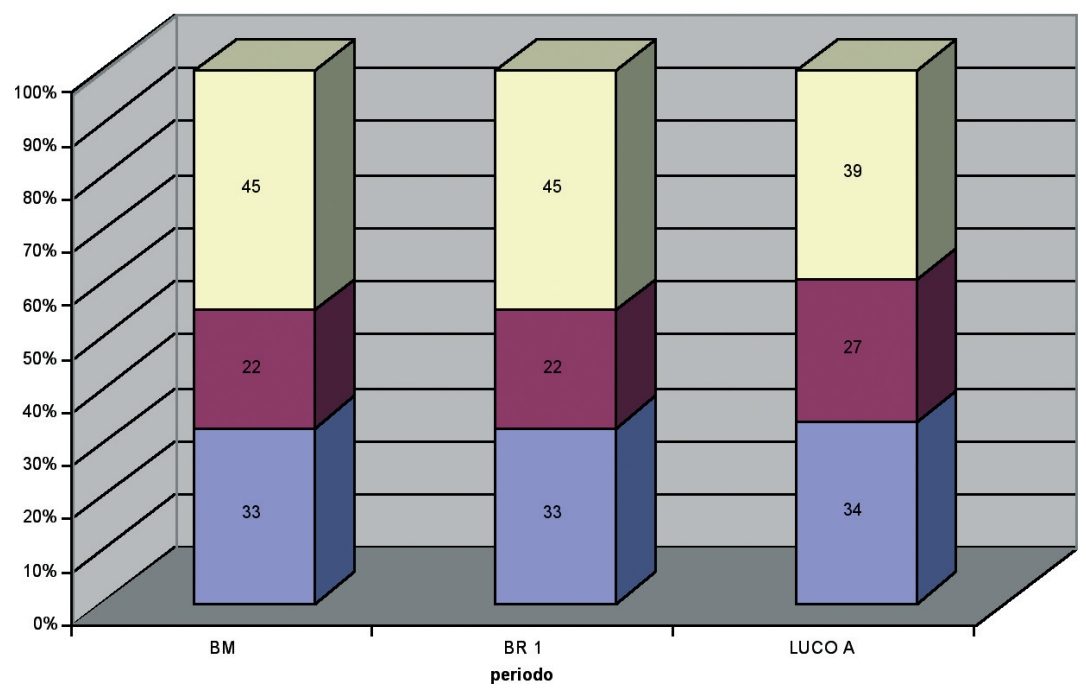

Fig. 6: Utilizzazione presunta del suolo nei siti dell'Alto Adige dal Bronzo medio al Bronzo finale (BM= Bronzo medio; BR: Bronzo recente)

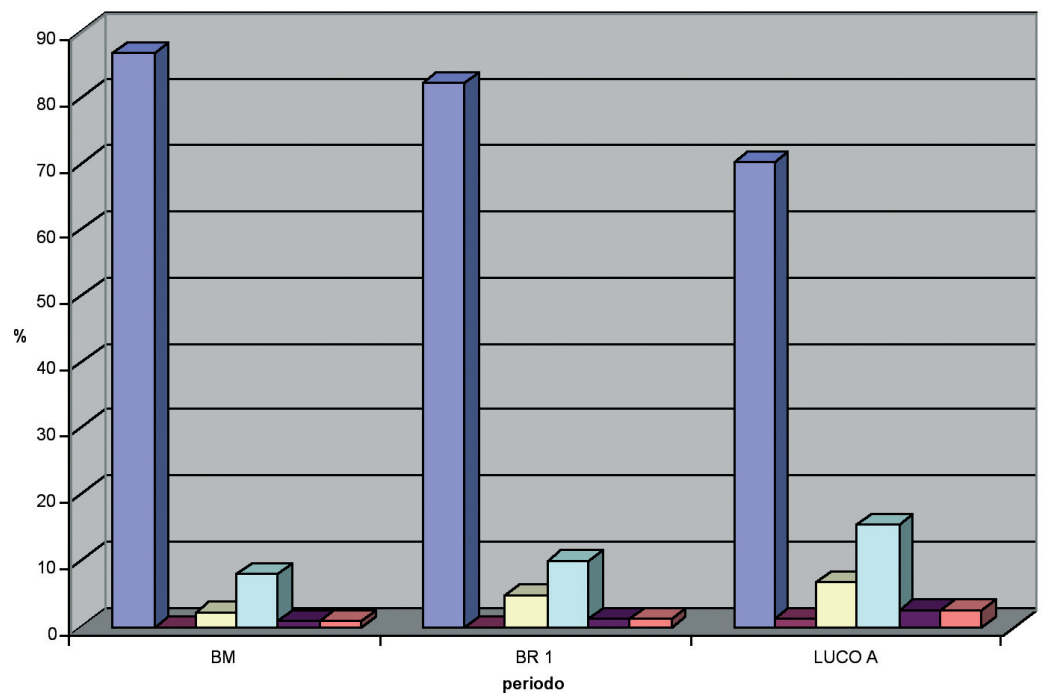

Fig. 7: Tipologia degli insediamenti dell'Alto Adige dal Bronzo medio al Bronzo finale ( $\mathrm{BM}=$ Bronzo medio; BR: Bronzo recente) 
D'altra parte, è ben noto, grazie agli studi condotti nel corso degli anni, da Ernst Preuschen e Lorenzo Dal RI ${ }^{18}$ e, più di recente, da Renato Perini, ${ }^{19}$ Franco Marzatico ed il personale scientifico del Deutsches Bergbaumuseum di Bochum, ${ }^{20}$ come le fasi avanzate dell'età del Bronzo vedano una notevole crescita delle attività di prospezione mineraria, estrazione del rame e ottenimento del metallo: già al Bronzo medio avanzato o recente appare databile per es. il complesso di forni a batteria rinvenuto a Favogna, ${ }^{21}$ sopra Cortaccia, nell'Alto Adige meridionale, ma non è questa la sede per affrontare l'argomento.

Elementi di un certo rilievo vengono pure dall'analisi delle caratteristiche topografiche degli insediamenti (Fig. 7): per quanto il modello del sito d'altura sia largamente maggioritario, è evidente che, nelle fasi più avanzate dell'età del Bronzo, si venga a stabilire una maggiore varietà di soluzioni insediative, con un significativo incremento di posizioni apparentemente poco o niente affatto difese (abitati su conoide, versante e fondovalle), che paiono avere una spiccata vocazione agricola. ${ }^{22}$ Il quadro provinciale presenta un certo contrasto con la situazione trentina, dove è riscontrabile un aumento dei siti d'altura, seppure non ai livelli dell'Alto Adige nel Bronzo antico e medio.

Nello specifico dei siti d'altura, durante l'età del Bronzo si osserva un aumento dell'altezza relativa degli abitati: avremmo, così, una situazione in cui gli insediamenti su rilievo, pur diminuiti di numero, manifestano una certa tendenza all'arroccamento. Di contro alla situazione del Bronzo antico-medio, in cui, sostanzialmente, l'unico modello è costituito dal sito d'altura, nel corso del Bronzo tardo la tipologia si polarizza, da un lato sull'abitato su rilievo, dall'altro sull'insediamento in posizione apparentemente non difesa.

Importanza per così dire epocale ha la scomparsa degli abitati in ambiente umido: gli unici due siti noti in tutta la regione, per il Bronzo finale, sembrano interpretabili come luoghi sacri, e dunque s'inseriscono nella problematica dei culti nelle o delle acque, trattati anche di recente in altra sede. ${ }^{23}$

${ }^{18}$ Cf. Preuschen 1973.

${ }^{19}$ Cf. Perini 1989.

${ }^{20}$ Cf. Cierny et al. 1998.

${ }^{21}$ Cf. Hauser/Nothdurfter 1986.

${ }^{22}$ Ciò può essere confermato dall'evidenza archeozoologica da Appiano-Siechenhaus (Bronzo recente-finale: RIEDEL 1985) e da Vadena-Laimburg (RIEDEL 2002), livelli inferiori (Bronzo finale), che mostra prevalenza dei bovini e buona percentuale di suini, per quanto questi ultimi si possano prestare a forme d'allevamento brado nelle aree umide. Considerazioni generali sull'inferenza archeozoologica nell'ermeneutica della funzione e del significato dei siti e dei sistemi insediativi in RIEDEL/TeCCHIATI 1999.

${ }^{23}$ Cf. Dal Ri/Tecchiati 2002; Di Pillo/Tecchiati 2003. 


\section{Strutture d'abitato: case e infrastrutture}

Considerati la carenza di siti scavati e l'insufficiente stato delle pubblicazioni, non sorprende che i dati sulle strutture interne ai siti siano particolarmente scarsi.

Prescindendo dai resti di singole strutture abitative, di solito assai labili, interessa, in questa sede, trattare l'impianto generale degli insediamenti e le testimonianze di infrastrutture.

Nel corso dell'età del Bronzo, specialmente a partire da una fase medio-recente, in effetti, acquistano particolare diffusione, sia in Trentino che in Alto Adige, gli insediamenti terrazzati, con abitazioni tendenzialmente omologhe disposte in maniera ordinata: questi siti testimoniano la coesione dei gruppi sociali, oltre alle loro capacità organizzative, che paiono ravvisabili anche nelle opere di fortificazione che, allo stato attuale delle conoscenze, compaiono, o almeno si affermano, a partire dal medesimo momento.

Per quanto in pratica solo nei casi di Varna-Nössing ${ }^{24}$, Badia-Sotćiastel ${ }^{25}$ e SludernoGanglegg ${ }^{26}$ si abbia la prova stratigrafica della pertinenza del muro di fortificazione (nei primi due abitati del tipo più semplice, a sbarramento del lato di accesso) all'età del Bronzo (fase media per Nössing e Sotćiastel, medio-recente per il Ganglegg), i siti che presentano, unicamente o quasi, tracce dell'età del Bronzo e resti di fortificazione sono molti di più: tra di essi, non pochi sono di ridottissime dimensioni (Nössing stesso è tra questi), sicché appare difficile immaginarli come siti egemoni.

Non mancano neppure, all'interno degli abitati del Bronzo recente-finale, opere di regimazione delle acque, piovane o di corsi d'acqua prossimali: resti interpretabili in questo senso sono stati rinvenuti per es. ad Appiano-Siechenhaus e a Terlano-Settequerce (via Steuer).

Un impianto abitativo piuttosto interessante è quello del sito, recentemente indagato, di Mals-Margun Egg, databile al Luco A, ${ }^{27}$ ove si ha attestazione di una serie di case disposte in maniera estremamente ordinata, cui s'affiancavano grandi fosse d'uso verosimilmente artigianale, così come appare documentato nel citato sito presso Appiano.

\footnotetext{
${ }^{24}$ Cf. TeCchiati 1998 b.

${ }^{25}$ Cf. TeCChiati 1998a.

${ }^{26} \mathrm{Cf}$. Gamper/Steiner 2000.

${ }^{27}$ Cf. Bombonato/Lunz 1991-1992.
} 
L'esistenza di opere infrastrutturali di discreto impegno solleva il problema della possibile strutturazione del paesaggio agrario: in particolare, la frequenza dei terrazzi abitativi rende oltremodo probabile l'apprestamento di manufatti consimili ad uso agricolo, come da tempo indiziato già almeno a partire dall'antica età del Bronzo, non solo in area atesina. D'altra parte, la straordinaria perizia nella lavorazione del legno, rivelata dagli scavi di Fiavè-Carera, può far supporre l'esistenza di opere di bonifica delle aree umide e dei fondivalle, forse anche solo allo scopo di garantirne la percorribilità. ${ }^{28}$

\section{Sistemi insediativi nella tarda età del Bronzo: due esempi}

Poiché la qualità del dato non permette di entrare nello specifico di ogni areale, $\mathrm{e}$ la morfologia della regione consiglia una certa cautela nell'applicazione di modelli desunti dalla geografia antropica, in questa sede ci si limiterà ad analizzare brevemente due comparti territoriali meglio noti alla ricerca scientifica: la conca di Bressanone e l'Oltradige.

La conca di Bressanone appare costellata da una serie di insediamenti, di dimensioni variabili, posti a quote differenti tutt'intorno al piano e sui terrazzi inferiori dell'Isarco: in questo comparto esiste una notevole continuità di occupazione, per lo meno tra il Bronzo antico e il recente, e in un caso si ha un insediamento (Albanbühel) che perdura, sebbene con dislocazioni minori, per tutto il Bronzo e il Ferro. Novità importante, nel periodo Luco A, nondimeno, è il sito del Kassianeum, sul terrazzo inferiore dell'Isarco, praticamernte in fondovalle, cui si connette il vasto abitato alle pendici del Pfeffersberg, purtroppo ancora poco noto. ${ }^{29}$ Di recente individuazione (scavi 2002-2003) è poi l'abitato del Bronzo finale di Via Castellano a Bressanone, quasi in riva alla Rienza (lato sinistro) nel quartiere di Stufles, con ricca documentazione strutturale e di cultura materiale..$^{30}$

L'esistenza di "grappoli" di insediamenti è testimoniata dal caso di Plabach, ove, sulla stessa unità orografica, insistono due abitati distinti e certamente coevi.

${ }^{28}$ Pensiamo ad opere (piste, sentieri) come quelle ben documentate nelle torbiere e paludi dell'Europa nord-occidentale.

${ }^{29}$ Cf. Rizzi/TeCchiati 2002. Una tesi di laurea sul sito è attualmente in corso a cura di A. Neri (relatore Prof. A. Bonardi) presso la Facoltà di Lettere e Filosofia dell'Università degli Studi di Parma.

${ }^{30} \mathrm{Cf}$. Parnigotto/Pisoni/Tecchiati (in stampa). 
Un tipico caso di comunità policentriche appare, con ogni probabilità, quello dell'Oltradige, in particolare nell'area del Comune (sparso ancora oggi) di Appiano: sui dossi morenici della zona si concentrano molti insediamenti contemporanei, quasi sempre di lunga durata (si pensi al caso del Putzer Gschleier), di dimensioni generalmente ridotte ed a brevissima distanza l'uno dall'altro.

\section{Sistemi insediativi nella tarda età del Bronzo: proposte interpretative}

L'elemento probabilmente di maggiore rilevanza, nel quadro insediativo della tarda età del Bronzo dell'area, è la comparsa di siti in posizione non rilevata, a svantaggio degli stanziamenti d'altura, i quali, ancorché sempre numericamente maggioritari, sembrano perdere importanza di fronte all'esplosione dei primi, che avranno una lunga tradizione per buona parte dell'età del Ferro (si pensi a Terlano-Settequerce o Vadena-Laimburg).

D'altra parte, i dati ricavabili dalle aree meglio note alla ricerca, lasciano supporre l'esistenza, forse anche più che nei momenti precedenti, di comunità di tipo "policentrico", ossia distribuite su diverse posizioni all'interno del rispettivo areale, forse come persistenza di antiche distinzioni tra gruppi parentelari, ma senza dubbio anche allo scopo di sfruttare in maniera capillare le risorse dell'ambiente e di assicurare un efficiente controllo del territorio.

Questo potrebbe spiegare il sorgere di insediamenti di dimensioni cospicue in situazioni morfologiche apparentemente poco difendibili, ${ }^{31}$ come, per contro, la presenza di siti di modesta o modestissima entità in posizioni arroccate e con resti di impianti fortificatori: durante il Bronzo tardo si viene a creare, in tal modo, un vero sistema insediativo costituito, per lo meno dove le condizioni lo consentivano, da abitati disposti in posizioni differenti, taluni anche temporanei, volti allo sfruttamento delle varie nicchie ecologiche e al controllo dei "confini" e delle vie di comunicazione.

In questo senso appare logico che i siti d'altura e quelli in quota fossero destinati alle pratiche pastorali e, in generale, ad attività di sfruttamento delle risorse "marginali" (silvicoltura, eventualmente estrazione mineraria), attività che peraltro, come detto, manifestano un'evidente intensificazione durante queste

\footnotetext{
${ }^{31}$ Non si può escludere, comunque, che siti come Vadena-Laimburg e Terlano-Settequerce fossero protetti a valle dallo stesso fiume Adige, che allora presentava un corso fortemente meandriforme (Coltorti/Dal Ri 1985).
} 
fasi, costituendo uno dei probabili fondamenti materiali delle élites; riconoscendo ai siti in piano una funzione eminentemente agricola e di partecipazione agli scambi.

Se quanto detto precedentemente forse spiega come abitati di fondovalle (o conoide) potessero essere difesi (attraverso il controllo costante degli accessi al territorio), rimane da capire quali circostanze abbiano spinto i gruppi sociali del tardo Bronzo a "colonizzare" le aree umide dei piani vallivi, o, altrimenti, abbiano consentito tale fenomeno: un'interpretazione in chiave ambientale (ad esempio una fase climatica asciutta) non sembra particolarmente convincente; preferibile, a nostro parere, è l'ipotesi che, nel periodo in discorso, abbiano avuto luogo i primi tentativi di controllare il corso dei fiumi, anche in considerazione di quanto evidenziato in precedenza, a proposito delle infrastrutture d'abitato.

Il concetto di "policentrico" non implica comunque una gerarchizzazione dei siti appartenenti alla medesima comunità: la documentazione disponibile, infatti, non sembra suggerire l'esistenza di una rigida differenziazione "politica" tra i siti, né essa appare necessaria al modello qui proposto. laddove la polarizzazione di posizioni, funzioni strategiche e vocazioni economiche potrebbe costituire solo la risposta ad un'esigenza di espansione e controllo territoriale.

\section{Bibliografia}

Alberti, A. et al.: Evidenze relative al X, IX, VIII sec. a. C. nell'ambito dell'alto bacino del fiume Adige (Cultura di Luco - Meluno), in: Atti del Convegno Tra Oriente e Occidente, Roma 2005 (in stampa).

Attardo, F./Banzi, E./Tecchiati, U. (eds.): Archäologie in Lajen. Archeologia a Laion, Bolzano 2004.

Bombonato, G./Lunz, R.: Un abitato preistorico presso Malles in Val Venosta, in: "Pagine di Ecologia”, XV, 42, 1991-1992, 63-66.

Cierny, J. et al.: Prehistoric Copper Metallurgy in the Southern Alpine Region, in: MoRdant, C./ Pernot, M./Rychner, V. (eds.), L'Atelier du bronzier en Europe du XXe au VIIIe siècle avant notre ère. Actes du colloque international Bronze '96, Paris 1998, 25-34.

Cocchi Genick, D. (ed.): L'età del Bronzo recente in Italia, Viareggio-Lucca 2004.

Coltorti, M./Dal Ri, L.: The human impact on the landscape: some examples from the Adige Valley, in: Malone, C./Stoddart, S. (eds.), Papers in Italian Archaeology IV. The Cambridge Conference. Part I. The Human Landscape, Oxford 1985, 105-134.

DAL RI, L.: Scavo di una capanna dell'età del Bronzo a San Paolo Appiano, in: Lunz 1990, op.cit., 77-86. 
DAL RI, L./TECCHIATI U.: I Gewässerfunde nella preistoria e protostoria dell'area alpina centromeridionale, in: Zemmer Plank, L. (ed.), Kult der Vorzeit in den Alpen. Opfergaben - Opferplätze - Opferbrauchtum / Culti nella Preistoria delle Alpi. Le offerte - i santuari - i riti, Bolzano 2002, 457-491.

Di Gennaro F./Tecchiati, U.: Insediamenti su rilievi, in: Cocchi, D. (ed.), L'antica età del bronzo in Italia, Firenze 1996, 229-245.

Di Pillo, M.: L'area atesina fra il Bronzo medio e l'inizio dell'età del Ferro, Tesi di Dottorato di Ricerca in Archeologia (Preistoria), VI ciclo, Università degli Studi di Roma-La Sapienza, Triennio Accademico 1991-1994.

Di Pillo, M.: Recensione a: Tecchiati 1998a, op.cit., in: "Ladinia”, XXIII, 1999, 267-278.

Di Pillo, M.: Settlement Patterns in the Upper Adige basin from the middle to the final Bronze age, in: Atti XXXIII Riun. Sc. I.I.P.P., Preistoria e Protostoria del Trentino Alto Adige/Südtirol in ricordo di Bernardino Bagolini, Firenze 2002, 105-115.

Di Pillo, M./Tecchiati, U.: Testimonianze di culti delle acque nel Trentino-Alto Adige durante l'età del bronzo. Inquadramento e spunti interpretativi, in: Negroni CATACchio, N. (ed.), Paesaggi d'acque. Ricerche e scavi, Milano 2003, 421-432.

GAMPER, P./STEInER, H.: Die Ausgrabungen 1998 und 1999 in der bronze- und eisenzeitlichen Siedlung am Ganglegg bei Schluderns. Stand der wissenschaftlichen Forschung, in: "Der Schlern", 74, 10, 2000, 601-663.

Hauser, L./Nothdurfter, H.: Bronzezeitliche Kupferschmelzhöfen in "Fennhals” über Kurtatsch, Bolzano 1986, 177-190.

LeITNER, W.: Eppan - St. Pauls, eine Siedlung der späten Bronzezeit. Ein Beitrag zur inneralpinen Laugen/Melaun Kultur, in: "Archaeologia Austriaca”, 72, 1988, 1-90.

LunZ, R. (ed.): Ur- und Frühgeschichte des Eppaner Raumes, Eppan 1990.

Marzatico, F.: Gli insediamenti di Dosso Alto e di Nomi Cef nel quadro della Recente età del Bronzo, in: "Annuario Storico della Valpolicella”, Fumane 1985-1986, 35-52.

Niederwanger, G./Tecchiati, U.: Acqua, Fuoco, Cielo. Seeberg: un luogo di roghi votivi di minatori della tarda età del bronzo nelle Alpi Sarentine. Catalogo della mostra, Bolzano 2000.

Parnigotto, I./Pisoni, L./Tecchiati, U.: Nuovi dati e riflessioni sul Bronzo finale nella conca di Bressanone (BZ): risultati della scavo di Via Castellano (campagne 2002-2003), Firenze (in stampa).

PerINI, R.: Area cultuale preistorica sulla Groa di Sopramonte (Trento), in: "Studi Trentini di Scienze Storiche", sez. II, LVIII, 1, 1979, 41-65.

PERINI, R.: Scavi archeologici nella zona palafitticola di Fiavé-Carera. Parte I, Trento 1984.

PerInI, R.: Scavi archeologici nella zona palafitticola di Fiavé-Carera. Parte II, Trento 1987.

PERINI, R.: Testimonianze di attività metallurgica dall'Eneolitico alle fasi finali dell'età del Bronzo in Trentino. Per Giuseppe Sebesta scritti e nota bio-bibliografica per il settantesimo compleanno, Trento 1989.

PerInI, R.: Scavi archeologici nella zona palafitticola di Fiavé-Carera. Parte III, Trento 1994.

Preuschen, E.: Estrazione mineraria dell'età del Bronzo nel Trentino, in: "Preistoria alpina", 9, 1973, 113-150.

Riedel, A.: Die Fauna einer bronzezeitlichen Siedlung bei Appiano (Südtirol), in: "Rivista di Archeologia", IX, 1985, 9-27. 
Riedel, A.: La fauna dell'insediamento protostorico di Vadena / Die Fauna der vorgeschichtlichen Siedlung von Vadena, Rovereto 2002.

Riedel, A./Tecchiati, U.: Settlements and economy in the Bronze and Iron Age in Trentino-South Tyrol. Notes for an archaeozoological model, in: "Preistoria Alpina", 35, 1999, 105-114.

Rizzi, G./Tecchiati, U.: Un insediamento della Cultura di Luco a Bressanone - Via Monte Ponente, in: Atti XXXIII riunione scientifica dell'I.I.P.P. Preistoria e Protostoria del Trentino Alto Adige/ Südtirol in ricordo di Bernardino Bagolini, Firenze 2002, 407-410.

SAlZANI, L.: Il sito protostorico di Custoza (Sommacampagna - Verona), in: "Padusa”, XXXII-XXXIII, 1996-1997, 7-46.

Tecchiati, U. (ed.): Sotćiastel. Un abitato fortificato dell'età del bronzo in Val Badia, San Martin de Tor/Bolzano 1998a.

TeCChiati, U.: Il "castelliere” Nössing: un insediamento d'altura dell'antica e media età del bronzo in Val d'Isarco (Bolzano), Tesi di Dottorato di ricerca in Archeologia: Insediamenti, Economia e Cultura (Preistorica, Greca, Romana, Medioevale), IX Ciclo, Consorzio Universitario di Pisa, Firenze e Siena 1998b.

Tecchiati, U.: Dinamiche insediative e gestione del territorio in Alto Adige tra la fine del III e la fine del I millennio a.C., in: Atti del Convegno di Sluderno (BZ) sugli abitati d'altura e i sistemi insediativi nella regione alpina centrale nell'età del Bronzo e del Ferro, Bolzano 2005 (in stampa).

\section{Resumé}

Chest contribut trata di sistems de insediament sciche esprescion de formes de organisazion dl teritore menudes entant 1 Bron tardif te Südtirol. Aldò de co che i stii de insediament se svilupeia entant 1 II milené dant Crist capéscen che i modiei de ocupazion y de gestion dl teritore se desferenzieia tres deplù, conseguenza chesta de na economia da paur y da paster dret efizienta y bona de emplenì fora ciamps produtifs desvalifs, per cie che reverda l'auteza y la ecologia, y che les sperses di insediamenc y i raions a chi che chestes é liedes cresc tres deplù. L fat che al vegne su tl Bron tardif insediamenc nia defenus y dlongia i rufs prinzipai, sceben che al n'en fossa nia mancé tl passé, mess gnì interpreté sot a la lum dles desposizions politich-teritoriales y de nezescités de control nueves di barac intra- y interculturai. L prozes de smendrament tres majer de insediamenc arjonj sie pont plu aut te momenc svilupés dl Bron tardif can che al se forma la cultura alpina zentrala de Laugen-Mellaun. 